Acta Crystallographica Section E

Structure Reports

Online

ISSN 1600-5368

Francesco Punzo, ${ }^{\mathrm{a}}{ }^{*}$ David J. Watkin, Jeroen Van Ameijde, Graeme Horne, ${ }^{c, d}$ George W. J. Fleet, ${ }^{\mathrm{c}}$ Mark R. Wormald ${ }^{\mathrm{e}}$ and Robert J. Nash ${ }^{\text {d }}$

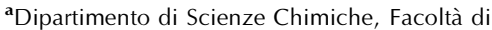
Farmacia, Università di Catania, Viale A. Doria 6, 95125 Catania, Italy, bepartment of Chemical Crystallography, Chemical Research Laboratory, Mansfield Road, Oxford OX1 3TA, England, 'Department of Organic Chemistry, Chemical Research Laboratory, Mansfield Road, Oxford OX1 3TA, England, ' ${ }^{\text {MNLPharma }}$

Limited, Institute of Grassland and

Environmental Research, Aberystwyth SY23 3EB, Dyfed, Wales, and ${ }^{\mathbf{e}}$ Glycobiology Institute, Department of Biochemistry, Oxford University, South Parks Road, Oxford OX1 3QU, England

₹ Visiting Scientist at the Department of Chemical Crystallography, Chemical Research Laboratory, Mansfield Road, Oxford OX1 3TA, England

Correspondence e-mail: fpunzo@unict.it

\author{
Key indicators \\ Single-crystal X-ray study \\ $T=120 \mathrm{~K}$ \\ Mean $\sigma(\mathrm{C}-\mathrm{C})=0.003 \AA$ \\ $R$ factor $=0.039$ \\ $w R$ factor $=0.091$ \\ Data-to-parameter ratio $=11.7$
}

For details of how these key indicators were automatically derived from the article, see http://journals.iucr.org/e.

\section{3,7,7a-Tri-epi-casuarine pentaacetate}

The relative stereochemistry at six contiguous centres in an analogue of the natural product casuarine, viz. 3,7,7a-tri-epicasuarine pentaacetate, $\mathrm{C}_{18} \mathrm{H}_{25} \mathrm{NO}_{10}$, has been established by an analysis of a crystalline pentaacetate.

\section{Comment}

The structure of casuarine, (1) (see scheme) (Nash et al., 1994), also isolated as its 6- $\alpha$-D-glucoside (Wormald et al., 1996), has been determined by X-ray crystallography. The crystal structure of 3-epi-casuarine, (2), has also been reported (Newton et al., 2004). Only two syntheses of casuarine have been published to date (Denmark \& Hurd, 2000; Izquierdo et al., 2005). Casuarine, with six contiguous stereogenic centres, is a potent $\alpha$-glucosidase inhibitor and is the most heavily oxygenated of the polyhydroxylated alkaloids which can be viewed as sugar mimics (Asano et al., 2000; Winchester \& Fleet, 1992). Synthetic studies on the epimers of casuarine are scant, and none of the stereoisomers reported significantly inhibited any glycosidase (Bell et al., 1997). Nonetheless, some casuarine analogues have promise as vaccine adjuvants and as potential candidates for viral disease and non-cytotoxic cancer therapies (Nash et al., 2004).

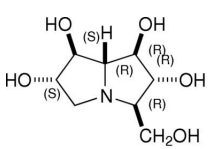

(I)

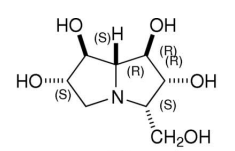

(2)

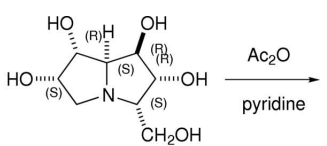

(3)

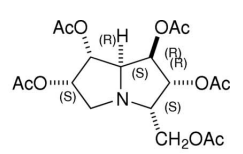

(4)
As part of a structure-activity investigation of the stereoisomers of casuarine, the tri-epi casuarine (3) was prepared by a route which did not define the relative configuration at two centres. Although (3) has not been crystallized, peracetylation by acetic anhydride in pyridine gave the crystalline pentaacetate, (4), the crystal structure of which is reported in this paper (Fig. 1 and Table 1).

This study firmly establishes the relative configuration at all six stereogenic centres. The absolute configuration of (4) is determined by the use of D-glucose as the starting material in the synthesis. A combination of crystal structures and NMR studies have established solid-state and solution conformations of a number of stereoisomers of the less oxygenated alexines (Wormald et al., 1998; Kato et al., 2003) which may be used to rationalize their biological activity. Similar structural studies on the stereoisomers of casuarine may permit the development of rationales for their novel biological activities. The crystal packing, represented in Fig. 2, highlights longrange interactions between the acetate fragments that are both non-polar, i.e. between methyl groups, and polar, i.e. between $\mathrm{O}$ atoms.
Received 25 January 2006 Accepted 31 January 2006
(C) 2006 International Union of Crystallography All rights reserved 


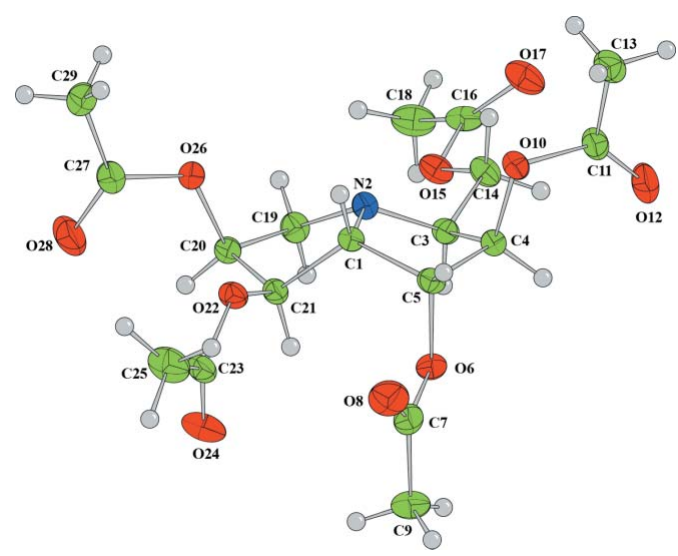

Figure 1

The molecular structure of (4), showing displacement ellipsoids drawn at the $50 \%$ probability level.

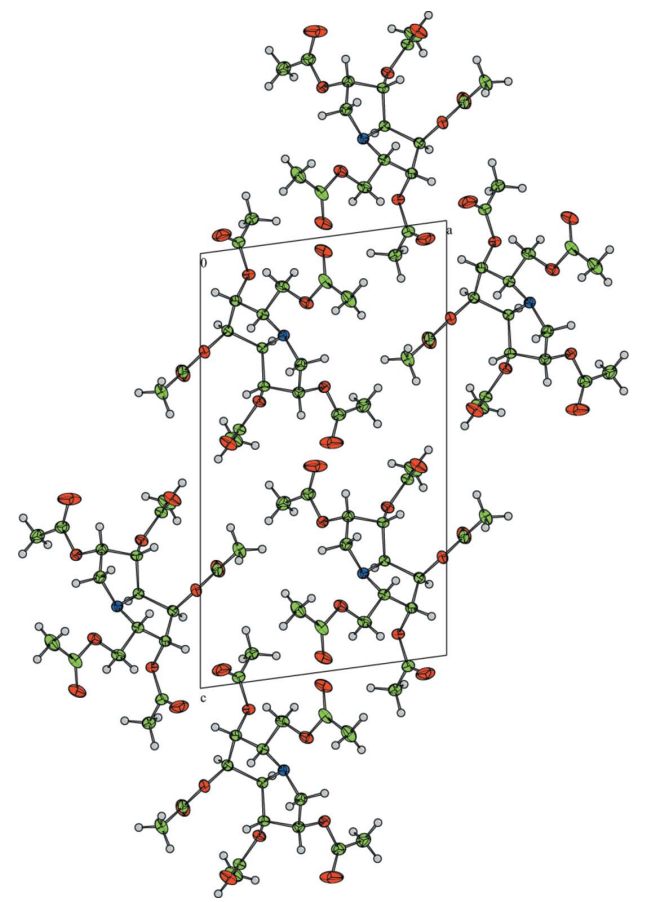

Figure 2

Packing diagram of (4), viewed down the $b$ axis.

\section{Experimental}

Compound (4) was crystallized by dissolving it in cyclohexane, adding ethanol (in an approximate ratio of 9:1), and allowing slow competitive evaporation of the two solvents until clear colourless crystals formed.

\section{Crystal data}

$\mathrm{C}_{18} \mathrm{H}_{25} \mathrm{NO}_{10}$

$M_{r}=415.40$

Monoclinic, $P 2_{1}$

$a=9.8357(3) \AA$

$b=5.9443(2) \AA$

$c=17.2146(6) \AA$

$\beta=97.6513(12)^{\circ}$

$V=997.51(6) \AA^{3}$

$Z=2$
$D_{x}=1.383 \mathrm{Mg} \mathrm{m}^{-3}$

Mo $K \alpha$ radiation

Cell parameters from 2338 reflections

$\theta=5-30^{\circ}$

$T=120 \mathrm{~K}$

Needle, colourless

$0.30 \times 0.10 \times 0.10 \mathrm{~mm}$ $\mu=0.11 \mathrm{~mm}^{-1}$
Data collection

Nonius KappaCCD diffractometer $\omega$ scans

Absorption correction: multi-scan (DENZO/SCALEPACK; Otwinowski \& Minor, 1997)

$T_{\min }=0.99, T_{\max }=0.99$

5106 measured reflections

\section{Refinement}

Refinement on $F^{2}$

$R\left[F^{2}>2 \sigma\left(F^{2}\right)\right]=0.039$

$w R\left(F^{2}\right)=0.091$

$S=0.94$

3067 reflections

263 parameters

$\mathrm{H}$-atom parameters constrained
3067 independent reflections 2513 reflections with $I>2 \sigma(I)$

$R_{\text {int }}=0.017$

$\theta_{\max }=30.0^{\circ}$

$h=-13 \rightarrow 13$

$k=-8 \rightarrow 7$

$l=-24 \rightarrow 24$

$w=1 /\left[\sigma^{2}\left(F^{2}\right)+(0.04 P)^{2}\right.$

$+0.2 P]$

where $P=\left[\max \left(F_{\mathrm{o}}{ }^{2}, 0\right)+2 F_{\mathrm{c}}{ }^{2}\right] / 3$

$(\Delta / \sigma)_{\max }<0.001$

$\Delta \rho_{\max }=0.33{\mathrm{e} \AA^{-3}}^{-3}$

$\Delta \rho_{\min }=-0.35$ e $\AA^{-3}$

Extinction correction: Larson (1970), equation 22

Extinction coefficient: $1.8(4) \times 10^{2}$

Table 1

Selected geometric parameters $\left(\AA{ }^{\circ}\right)$.

\begin{tabular}{|c|c|c|c|}
\hline $\mathrm{C} 1-\mathrm{N} 2$ & $1.486(2)$ & $\mathrm{C} 11-\mathrm{C} 13$ & $1.491(3)$ \\
\hline $\mathrm{C} 1-\mathrm{C} 5$ & $1.540(3)$ & $\mathrm{C} 14-\mathrm{O} 15$ & $1.448(2)$ \\
\hline $\mathrm{C} 1-\mathrm{C} 21$ & $1.517(3)$ & $\mathrm{O} 15-\mathrm{C} 16$ & $1.351(2)$ \\
\hline $\mathrm{N} 2-\mathrm{C} 3$ & $1.466(2)$ & $\mathrm{C} 16-\mathrm{O} 17$ & $1.208(3)$ \\
\hline $\mathrm{N} 2-\mathrm{C} 19$ & $1.477(2)$ & $\mathrm{C} 16-\mathrm{C} 18$ & $1.483(3)$ \\
\hline $\mathrm{C} 3-\mathrm{C} 4$ & $1.528(3)$ & $\mathrm{C} 19-\mathrm{C} 20$ & $1.513(3)$ \\
\hline $\mathrm{C} 3-\mathrm{C} 14$ & $1.508(3)$ & $\mathrm{C} 20-\mathrm{C} 21$ & $1.523(3)$ \\
\hline $\mathrm{C} 4-\mathrm{C} 5$ & $1.525(3)$ & $\mathrm{C} 20-\mathrm{O} 26$ & $1.456(2)$ \\
\hline $\mathrm{C} 4-\mathrm{O} 10$ & $1.448(2)$ & $\mathrm{C} 21-\mathrm{O} 22$ & $1.432(2)$ \\
\hline $\mathrm{C} 5-\mathrm{O} 6$ & $1.453(2)$ & $\mathrm{O} 22-\mathrm{C} 23$ & $1.363(2)$ \\
\hline $\mathrm{O} 6-\mathrm{C} 7$ & $1.357(3)$ & $\mathrm{C} 23-\mathrm{O} 24$ & 1.197 (3) \\
\hline $\mathrm{C} 7-\mathrm{O} 8$ & $1.197(3)$ & $\mathrm{C} 23-\mathrm{C} 25$ & $1.490(3)$ \\
\hline $\mathrm{C} 7-\mathrm{C} 9$ & $1.493(4)$ & $\mathrm{O} 26-\mathrm{C} 27$ & $1.355(2)$ \\
\hline $\mathrm{O} 10-\mathrm{C} 11$ & $1.355(2)$ & $\mathrm{C} 27-\mathrm{O} 28$ & $1.193(3)$ \\
\hline $\mathrm{C} 11-\mathrm{O} 12$ & $1.200(3)$ & $\mathrm{C} 27-\mathrm{C} 29$ & $1.482(3)$ \\
\hline $\mathrm{N} 2-\mathrm{C} 1-\mathrm{C} 5$ & $106.11(15)$ & $\mathrm{O} 12-\mathrm{C} 11-\mathrm{C} 13$ & 126.35 (19) \\
\hline $\mathrm{N} 2-\mathrm{C} 1-\mathrm{C} 21$ & $104.58(15)$ & $\mathrm{C} 3-\mathrm{C} 14-\mathrm{O} 15$ & $107.24(15)$ \\
\hline $\mathrm{C} 5-\mathrm{C} 1-\mathrm{C} 21$ & $118.30(16)$ & $\mathrm{C} 14-\mathrm{O} 15-\mathrm{C} 16$ & $114.62(16)$ \\
\hline $\mathrm{C} 1-\mathrm{N} 2-\mathrm{C} 3$ & 108.97 (14) & $\mathrm{O} 15-\mathrm{C} 16-\mathrm{O} 17$ & $122.2(2)$ \\
\hline $\mathrm{C} 1-\mathrm{N} 2-\mathrm{C} 19$ & $108.76(15)$ & $\mathrm{O} 15-\mathrm{C} 16-\mathrm{C} 18$ & $112.17(18)$ \\
\hline $\mathrm{C} 3-\mathrm{N} 2-\mathrm{C} 19$ & $116.67(16)$ & $\mathrm{O} 17-\mathrm{C} 16-\mathrm{C} 18$ & $125.6(2)$ \\
\hline $\mathrm{N} 2-\mathrm{C} 3-\mathrm{C} 4$ & $103.27(16)$ & $\mathrm{N} 2-\mathrm{C} 19-\mathrm{C} 20$ & $105.06(17)$ \\
\hline $\mathrm{N} 2-\mathrm{C} 3-\mathrm{C} 14$ & $113.92(16)$ & $\mathrm{C} 19-\mathrm{C} 20-\mathrm{C} 21$ & $101.52(15)$ \\
\hline $\mathrm{C} 4-\mathrm{C} 3-\mathrm{C} 14$ & $112.75(15)$ & $\mathrm{C} 19-\mathrm{C} 20-\mathrm{O} 26$ & $108.48(16)$ \\
\hline $\mathrm{C} 3-\mathrm{C} 4-\mathrm{C} 5$ & $103.33(15)$ & $\mathrm{C} 21-\mathrm{C} 20-\mathrm{O} 26$ & $109.03(16)$ \\
\hline $\mathrm{C} 3-\mathrm{C} 4-\mathrm{O} 10$ & $111.18(15)$ & $\mathrm{C} 20-\mathrm{C} 21-\mathrm{C} 1$ & $103.69(16)$ \\
\hline $\mathrm{C} 5-\mathrm{C} 4-\mathrm{O} 10$ & $106.33(16)$ & $\mathrm{C} 20-\mathrm{C} 21-\mathrm{O} 22$ & $114.18(16)$ \\
\hline $\mathrm{C} 1-\mathrm{C} 5-\mathrm{C} 4$ & $103.08(15)$ & $\mathrm{C} 1-\mathrm{C} 21-\mathrm{O} 22$ & $110.11(16)$ \\
\hline $\mathrm{C} 1-\mathrm{C} 5-\mathrm{O} 6$ & 111.65 (15) & $\mathrm{C} 21-\mathrm{O} 22-\mathrm{C} 23$ & $117.04(16)$ \\
\hline $\mathrm{C} 4-\mathrm{C} 5-\mathrm{O} 6$ & $104.60(16)$ & $\mathrm{O} 22-\mathrm{C} 23-\mathrm{O} 24$ & $122.9(2)$ \\
\hline $\mathrm{C} 5-\mathrm{O} 6-\mathrm{C} 7$ & 116.67 (17) & $\mathrm{O} 22-\mathrm{C} 23-\mathrm{C} 25$ & 110.27 (19) \\
\hline $\mathrm{O} 6-\mathrm{C} 7-\mathrm{O} 8$ & $123.5(2)$ & $\mathrm{O} 24-\mathrm{C} 23-\mathrm{C} 25$ & $126.8(2)$ \\
\hline $\mathrm{O} 6-\mathrm{C} 7-\mathrm{C} 9$ & $110.9(2)$ & $\mathrm{C} 20-\mathrm{O} 26-\mathrm{C} 27$ & $117.07(16)$ \\
\hline $\mathrm{O} 8-\mathrm{C} 7-\mathrm{C} 9$ & $125.6(2)$ & $\mathrm{O} 26-\mathrm{C} 27-\mathrm{O} 28$ & $122.8(2)$ \\
\hline $\mathrm{C} 4-\mathrm{O} 10-\mathrm{C} 11$ & $116.98(16)$ & $\mathrm{O} 26-\mathrm{C} 27-\mathrm{C} 29$ & $112.16(18)$ \\
\hline $\mathrm{O} 10-\mathrm{C} 11-\mathrm{O} 12$ & $122.9(2)$ & $\mathrm{O} 28-\mathrm{C} 27-\mathrm{C} 29$ & $125.1(2)$ \\
\hline $\mathrm{O} 10-\mathrm{C} 11-\mathrm{C} 13$ & $110.71(19)$ & & \\
\hline
\end{tabular}

In the absence of significant anomalous scattering effects, Friedel pairs were merged, and the absolute configuration was assigned from the known configuration of the starting material. $\mathrm{H}$ atoms were seen in a difference density synthesis. Those attached to $\mathrm{C}$ atoms were repositioned geometrically. The $\mathrm{H}$ atoms were initially refined with soft restraints on the bond lengths and angles to regularize their geometry, after which they were included with riding constraints, with $\mathrm{C}-\mathrm{H}=0.93-0.98 \AA$ and with $U_{\text {iso }}(\mathrm{H})$ values in the range $1.2-1.5 U_{\text {eq }}$ of the carrier atom. 
Data collection: COLLECT (Nonius, 2001); cell refinement and data reduction: DENZO/SCALEPACK (Otwinowski \& Minor, 1997); program(s) used to solve structure: SIR92 (Altomare et al., 1994); program(s) used to refine structure: CRYSTALS (Betteridge et al., 2003); molecular graphics: CAMERON (Watkin et al., 1996); software used to prepare material for publication: CRYSTALS.

\section{References}

Altomare, A., Cascarano, G., Giacovazzo G., Guagliardi A., Burla M. C., Polidori, G. \& Camalli, M. (1994). J. Appl. Cryst. 27, 435.

Asano, N., Nash, R. J., Molyneux, R. J. \& Fleet, G. W. J. (2000). Tetrahedron Asymmetry, 11, 1645-1680.

Bell, A. A., Pickering, L., Watson, A. A., Nash, R. J., Pan, Y. T., Elbein, A. D. \& Fleet, G. W. J. (1997). Tetrahedron Lett. 38, 5869-5872.

Betteridge, P. W., Carruthers, J. R., Cooper, R. I., Prout, K. \& Watkin, D. J. (2003). J. Appl. Cryst. 36, 1487.

Denmark, S. E. \& Hurd, A. R. (2000). J. Org. Chem. 65, 2875-2886.

Izquierdo, I., Plaza, M. T., Juan, A. \& Tamayo, J. A. (2005). Tetrahedron, 61, $6527-6533$.
Kato, A., Kano, E., Adachi, I., Molyneux, R. J., Watson, A. A., Nash, R. J., Fleet, G. W. J., Wormald, M. R., Kizu, H., Ikeda, K. \& Asano, N. (2003). Tetrahedron Asymmetry, 14, 325-331.

Larson, A. C. (1970). Crystallographic Computing, edited by F. R. Ahmed, S. R. Hall \& C. P. Huber, pp. 291-294. Copenhagen: Munksgaard.

Nash, R. J., Thomas, P. I., Waigh, R. D., Fleet, G. W. J., Wormald, M. R., Lilley, P. M. Q. \& Watkin, D. J. (1994). Tetrahedron Lett. 35, 7849-7852.

Nash, R. J., Watson, A. A. \& Evinson, E. L. (2004). PCT Int. Appl. 2004, WO2004064715.

Newton, C., van Ameijde, J., Fleet, G. W. J., Nash, R. J. \& Watkin, D. J. (2004). Acta Cryst. E60, o1463-o1464.

Nonius (2001). COLLECT. Nonius BV, Delft, The Netherlands.

Otwinowski, Z. \& Minor, W. (1997). Methods in Enzymology, Vol. 276, Macromolecular Crystallography, Part A, edited by C. W. Carter Jr \& R. M. Sweet, pp. 307-326. New York: Academic Press.

Watkin, D. J., Prout, C. K. \& Pearce, L. J. (1996). CAMERON. Chemical Crystallography Laboratory, Oxford, England.

Winchester, B. \& Fleet, G. W. J. (1992). Glycobiology, 2, 199-210.

Wormald, M. R., Nash, R. J., Hrnicar, P., White, J. D., Molyneux, R. J. \& Fleet, G. W. J. (1998). Tetrahedron Asymmetry, 9, 2549-2558.

Wormald, M. R., Nash, R. J., Watson, A. A., Bhadoria, B. K., Langford, R., Sim, M. \& Fleet, G. W. J. (1996). Carbohydr. Lett. 2, 169-174. 


\section{supporting information}

Acta Cryst. (2006). E62, o928-0930 [https://doi.org/10.1107/S1600536806003539]

\section{3,7,7a-Tri-epi-casuarine pentaacetate}

Francesco Punzo, David J. Watkin, Jeroen Van Ameijde, Graeme Horne, George W. J. Fleet, Mark R. Wormald and Robert J. Nash

3,7,7a-triepi-Casuarine pentaacetate

Crystal data

$\mathrm{C}_{18} \mathrm{H}_{25} \mathrm{NO}_{10}$

$M_{r}=415.40$

Monoclinic, $P 2_{1}$

Hall symbol: P $2 \mathrm{yb}$

$a=9.8357(3) \AA$

$b=5.9443(2) \AA$

$c=17.2146(6) \AA$

$\beta=97.6513(12)^{\circ}$

$V=997.51(6) \AA^{3}$

$Z=2$

\section{Data collection}

Nonius KappaCCD diffractometer

Graphite monochromator

$\omega$ scans

Absorption correction: multi-scan

(DENZO/SCALEPACK; Otwinowski \& Minor, 1997)

$T_{\min }=0.99, T_{\max }=0.99$

\section{Refinement}

Refinement on $F^{2}$

Least-squares matrix: full

$R\left[F^{2}>2 \sigma\left(F^{2}\right)\right]=0.039$

$w R\left(F^{2}\right)=0.091$

$S=0.94$

3067 reflections

263 parameters

1 restraint

Primary atom site location: structure-invariant direct methods
$F(000)=440$

$D_{\mathrm{x}}=1.383 \mathrm{Mg} \mathrm{m}^{-3}$

Mo $K \alpha$ radiation, $\lambda=0.71073 \AA$

Cell parameters from 2338 reflections

$\theta=5-30^{\circ}$

$\mu=0.11 \mathrm{~mm}^{-1}$

$T=120 \mathrm{~K}$

Plate, colourless

$0.30 \times 0.10 \times 0.10 \mathrm{~mm}$

5106 measured reflections

3067 independent reflections

2513 reflections with $I>2 \sigma(I)$

$R_{\text {int }}=0.017$

$\theta_{\text {max }}=30.0^{\circ}, \theta_{\text {min }}=5.1^{\circ}$

$h=-13 \rightarrow 13$

$k=-8 \rightarrow 7$

$l=-24 \rightarrow 24$

Hydrogen site location: inferred from neighbouring sites

$\mathrm{H}$-atom parameters constrained

$w=1 /\left[\sigma^{2}\left(F^{2}\right)+(0.04 P)^{2}+0.2 P\right]$, where $P=\left[\max \left(F_{\mathrm{o}}^{2}, 0\right)+2 F_{\mathrm{c}}^{2}\right] / 3$

$(\Delta / \sigma)_{\max }<0.001$

$\Delta \rho_{\max }=0.33$ e $\AA^{-3}$

$\Delta \rho_{\min }=-0.35$ e $\AA^{-3}$

Extinction correction: Larson (1970), equation 22

Extinction coefficient: 180 (40)

Fractional atomic coordinates and isotropic or equivalent isotropic displacement parameters $\left(\AA^{2}\right)$

\begin{tabular}{lllll}
\hline & $x$ & $y$ & $z$ & $U_{\mathrm{iso}} * / U_{\text {eq }}$ \\
\hline $\mathrm{C} 1$ & $0.25143(19)$ & $0.1388(3)$ & $0.23642(10)$ & 0.0187
\end{tabular}




\begin{tabular}{|c|c|c|c|c|}
\hline $\mathrm{N} 2$ & $0.33807(16)$ & $0.3266(3)$ & $0.21412(9)$ & 0.0193 \\
\hline $\mathrm{C} 3$ & $0.2539(2)$ & $0.4752(3)$ & $0.15921(11)$ & 0.0201 \\
\hline $\mathrm{C} 4$ & 0.1427 (2) & $0.3181(4)$ & $0.11951(11)$ & 0.0219 \\
\hline $\mathrm{C} 5$ & 0.1107 (2) & 0.1667 (4) & $0.18624(11)$ & 0.0212 \\
\hline O6 & $0.01706(14)$ & $0.2978(3)$ & $0.22668(8)$ & 0.0255 \\
\hline $\mathrm{C} 7$ & $-0.0695(2)$ & $0.1806(5)$ & $0.26674(13)$ & 0.0297 \\
\hline $\mathrm{O} 8$ & $-0.07114(18)$ & $-0.0205(3)$ & $0.27014(10)$ & 0.0399 \\
\hline C9 & $-0.1587(2)$ & $0.3359(5)$ & $0.30573(15)$ & 0.0419 \\
\hline $\mathrm{O} 10$ & $0.19617(14)$ & $0.1727(3)$ & $0.06328(7)$ & 0.0237 \\
\hline C11 & $0.1557(2)$ & $0.2161(4)$ & $-0.01361(12)$ & 0.0286 \\
\hline $\mathrm{O} 12$ & $0.0866(2)$ & $0.3756(4)$ & $-0.03595(9)$ & 0.0480 \\
\hline $\mathrm{C} 13$ & $0.2093(2)$ & $0.0416(4)$ & $-0.06385(12)$ & 0.0333 \\
\hline $\mathrm{C} 14$ & $0.3328(2)$ & $0.5918(4)$ & $0.10152(12)$ & 0.0252 \\
\hline $\mathrm{O} 15$ & $0.42996(15)$ & $0.7417(3)$ & $0.14594(8)$ & 0.0262 \\
\hline $\mathrm{C} 16$ & $0.5080(2)$ & $0.8625(4)$ & $0.10221(13)$ & 0.0279 \\
\hline $\mathrm{O} 17$ & $0.49969(18)$ & $0.8407(3)$ & $0.03195(9)$ & 0.0368 \\
\hline $\mathrm{C} 18$ & $0.6021(3)$ & $1.0182(4)$ & $0.15052(15)$ & 0.0370 \\
\hline C19 & $0.4086(2)$ & $0.4316(4)$ & $0.28622(11)$ & 0.0240 \\
\hline $\mathrm{C} 20$ & 0.3997 (2) & $0.2597(3)$ & $0.35015(11)$ & 0.0217 \\
\hline $\mathrm{C} 21$ & $0.2588(2)$ & $0.1574(3)$ & $0.32480(11)$ & 0.0197 \\
\hline $\mathrm{O} 22$ & $0.23933(14)$ & $-0.0592(2)$ & $0.35803(8)$ & 0.0226 \\
\hline $\mathrm{C} 23$ & $0.1593(2)$ & $-0.0686(4)$ & $0.41678(12)$ & 0.0253 \\
\hline $\mathrm{O} 24$ & $0.11413(18)$ & $0.0956(3)$ & $0.44445(10)$ & 0.0387 \\
\hline $\mathrm{C} 25$ & $0.1360(3)$ & $-0.3071(4)$ & $0.43825(15)$ & 0.0405 \\
\hline $\mathrm{O} 26$ & $0.50414(14)$ & $0.0887(3)$ & $0.34492(8)$ & 0.0244 \\
\hline $\mathrm{C} 27$ & $0.5616(2)$ & $-0.0068(4)$ & $0.41296(12)$ & 0.0293 \\
\hline $\mathrm{O} 28$ & $0.5325(2)$ & $0.0480(4)$ & $0.47537(9)$ & 0.0593 \\
\hline $\mathrm{C} 29$ & $0.6623(2)$ & $-0.1838(4)$ & $0.39999(13)$ & 0.0326 \\
\hline H11 & 0.2925 & -0.0032 & 0.2234 & $0.0223^{*}$ \\
\hline H31 & 0.2060 & 0.5904 & 0.1875 & $0.0248^{*}$ \\
\hline $\mathrm{H} 41$ & 0.0609 & 0.4045 & 0.0941 & $0.0275^{*}$ \\
\hline H51 & 0.0706 & 0.0200 & 0.1689 & $0.0255^{*}$ \\
\hline H91 & -0.2507 & 0.2740 & 0.3010 & $0.0636^{*}$ \\
\hline H92 & -0.1571 & 0.4835 & 0.2819 & $0.0633^{*}$ \\
\hline H93 & -0.1231 & 0.3461 & 0.3599 & $0.0636^{*}$ \\
\hline H131 & 0.1851 & 0.0841 & -0.1188 & $0.0471^{*}$ \\
\hline H132 & 0.1720 & -0.1038 & -0.0543 & $0.0467^{*}$ \\
\hline H133 & 0.3078 & 0.0384 & -0.0515 & $0.0471^{*}$ \\
\hline H141 & 0.2686 & 0.6793 & 0.0640 & $0.0318^{*}$ \\
\hline H142 & 0.3809 & 0.4833 & 0.0725 & $0.0319^{*}$ \\
\hline H181 & 0.6624 & 1.0880 & 0.1172 & $0.0567^{*}$ \\
\hline H182 & 0.5493 & 1.1306 & 0.1729 & $0.0568^{*}$ \\
\hline H183 & 0.6548 & 0.9355 & 0.1916 & $0.0567^{*}$ \\
\hline H191 & 0.3629 & 0.5702 & 0.2997 & $0.0295^{*}$ \\
\hline H192 & 0.5052 & 0.4634 & 0.2795 & $0.0288^{*}$ \\
\hline H201 & 0.4091 & 0.3228 & 0.4030 & $0.0262^{*}$ \\
\hline H211 & 0.1877 & 0.2619 & 0.3382 & $0.0231^{*}$ \\
\hline H251 & 0.0736 & -0.3105 & 0.4783 & $0.0641^{*}$ \\
\hline
\end{tabular}




\begin{tabular}{|c|c|c|c|c|}
\hline H252 & 0.0947 & -0.3875 & 0.3925 & $0.0643 *$ \\
\hline $\mathrm{H} 253$ & 0.2224 & -0.3763 & 0.4583 & $0.0642 *$ \\
\hline H291 & 0.7195 & -0.2230 & 0.4466 & $0.0486 *$ \\
\hline H292 & 0.6200 & -0.3170 & 0.3772 & $0.0491 *$ \\
\hline H293 & 0.7217 & -0.1292 & 0.3640 & $0.0489 *$ \\
\hline
\end{tabular}

Atomic displacement parameters $\left(\AA^{2}\right)$

\begin{tabular}{|c|c|c|c|c|c|c|}
\hline & $U^{11}$ & $U^{22}$ & $U^{33}$ & $U^{12}$ & $U^{13}$ & $U^{23}$ \\
\hline $\mathrm{C} 1$ & $0.0188(9)$ & $0.0192(9)$ & $0.0185(9)$ & $-0.0001(7)$ & $0.0039(7)$ & $-0.0001(7)$ \\
\hline N2 & $0.0206(8)$ & $0.0185(8)$ & $0.0186(7)$ & $-0.0027(7)$ & $0.0019(6)$ & $-0.0008(7)$ \\
\hline $\mathrm{C} 3$ & $0.0225(10)$ & $0.0193(9)$ & $0.0190(9)$ & $0.0011(8)$ & $0.0046(8)$ & $0.0002(8)$ \\
\hline $\mathrm{C} 4$ & $0.0223(10)$ & $0.0254(10)$ & $0.0179(9)$ & $0.0034(9)$ & $0.0027(7)$ & $0.0003(8)$ \\
\hline $\mathrm{C} 5$ & $0.0206(9)$ & $0.0238(9)$ & $0.0200(9)$ & $-0.0014(8)$ & $0.0050(7)$ & $-0.0014(8)$ \\
\hline O6 & $0.0186(7)$ & $0.0320(8)$ & $0.0268(7)$ & $0.0007(6)$ & $0.0061(6)$ & $0.0013(6)$ \\
\hline $\mathrm{C} 7$ & $0.0168(10)$ & $0.0447(14)$ & $0.0277(11)$ & $-0.0037(10)$ & $0.0028(8)$ & $0.0057(11)$ \\
\hline $\mathrm{O} 8$ & $0.0318(9)$ & $0.0436(10)$ & $0.0464(10)$ & $-0.0063(8)$ & $0.0132(8)$ & $0.0078(9)$ \\
\hline C9 & $0.0269(12)$ & $0.0608(18)$ & $0.0406(13)$ & $0.0054(13)$ & $0.0141(10)$ & $0.0000(14)$ \\
\hline $\mathrm{O} 10$ & $0.0255(7)$ & $0.0282(7)$ & $0.0175(6)$ & $0.0002(6)$ & $0.0028(5)$ & $-0.0013(6)$ \\
\hline $\mathrm{C} 11$ & $0.0257(11)$ & $0.0404(13)$ & $0.0190(10)$ & $-0.0031(10)$ & $0.0007(8)$ & $0.0007(9)$ \\
\hline $\mathrm{O} 12$ & $0.0587(12)$ & $0.0609(13)$ & $0.0228(8)$ & $0.0217(11)$ & $-0.0005(8)$ & $0.0080(9)$ \\
\hline C13 & $0.0285(11)$ & $0.0487(15)$ & $0.0228(10)$ & $-0.0068(11)$ & $0.0028(9)$ & $-0.0075(11)$ \\
\hline C14 & $0.0314(11)$ & $0.0233(10)$ & $0.0219(10)$ & $-0.0036(9)$ & $0.0072(8)$ & $0.0001(9)$ \\
\hline $\mathrm{O} 15$ & $0.0310(8)$ & $0.0242(7)$ & $0.0251(7)$ & -0.0069 (6) & $0.0099(6)$ & $-0.0022(6)$ \\
\hline $\mathrm{C} 16$ & $0.0286(11)$ & $0.0220(10)$ & $0.0365(12)$ & 0.0012 (9) & 0.0169 (9) & $0.0012(9)$ \\
\hline $\mathrm{O} 17$ & $0.0489(10)$ & $0.0343(9)$ & $0.0309(8)$ & $-0.0048(8)$ & $0.0194(7)$ & $0.0041(8)$ \\
\hline $\mathrm{C} 18$ & $0.0356(13)$ & $0.0320(12)$ & 0.0469 (14) & $-0.0081(11)$ & $0.0185(11)$ & $-0.0050(11)$ \\
\hline C19 & $0.0242(10)$ & $0.0239(10)$ & $0.0234(10)$ & $-0.0037(8)$ & $0.0015(8)$ & $-0.0019(8)$ \\
\hline $\mathrm{C} 20$ & $0.0230(10)$ & $0.0223(9)$ & $0.0196(9)$ & $0.0028(8)$ & $0.0022(8)$ & $-0.0021(8)$ \\
\hline $\mathrm{C} 21$ & $0.0230(9)$ & $0.0193(9)$ & $0.0170(9)$ & $0.0023(8)$ & $0.0038(7)$ & $0.0011(8)$ \\
\hline $\mathrm{O} 22$ & $0.0265(8)$ & $0.0212(7)$ & $0.0214(7)$ & 0.0015 & $0.0079(6)$ & $0.0033(6)$ \\
\hline $\mathrm{C} 23$ & $0.0253(10)$ & $0.0322(12)$ & $0.0193(9)$ & $-0.0023(9)$ & $0.0066(8)$ & $0.0013(9)$ \\
\hline $\mathrm{O} 24$ & $0.0484(10)$ & $0.0351(9)$ & $0.0377(9)$ & $-0.0026(9)$ & $0.0239(8)$ & $-0.0063(8)$ \\
\hline $\mathrm{C} 25$ & $0.0505(16)$ & $0.0342(13)$ & 0.0408 (14) & $-0.0034(12)$ & $0.0212(12)$ & $0.0090(11)$ \\
\hline $\mathrm{O} 26$ & $0.0220(7)$ & $0.0304(8)$ & $0.0203(7)$ & $0.0037(7)$ & $0.0011(6)$ & $-0.0005(6)$ \\
\hline $\mathrm{C} 27$ & $0.0324(12)$ & $0.0323(11)$ & $0.0229(10)$ & $0.0036(10)$ & $0.0030(9)$ & $0.0032(9)$ \\
\hline $\mathrm{O} 28$ & $0.0895(16)$ & $0.0656(15)$ & $0.0238(9)$ & 0.0433 (13) & $0.0116(9)$ & 0.0103 (9) \\
\hline $\mathrm{C} 29$ & $0.0326(12)$ & $0.0351(12)$ & $0.0292(11)$ & $0.0086(11)$ & $0.0001(9)$ & $0.0005(10)$ \\
\hline
\end{tabular}

Geometric parameters $\left(\AA,{ }^{\circ}\right)$

\begin{tabular}{llll}
\hline $\mathrm{C} 1-\mathrm{N} 2$ & $1.486(2)$ & $\mathrm{C} 14-\mathrm{H} 142$ & 0.975 \\
$\mathrm{C} 1-\mathrm{C} 5$ & $1.540(3)$ & $\mathrm{O} 15-\mathrm{C} 16$ & $1.351(2)$ \\
$\mathrm{C} 1-\mathrm{C} 21$ & $1.517(3)$ & $\mathrm{C} 16-\mathrm{O} 17$ & $1.208(3)$ \\
$\mathrm{C} 1-\mathrm{H} 11$ & $\mathrm{C} 16-\mathrm{C} 18$ & $1.483(3)$ \\
$\mathrm{N} 2-\mathrm{C} 3$ & 0.974 & $\mathrm{C} 18-\mathrm{H} 181$ & 0.972 \\
$\mathrm{~N} 2-\mathrm{C} 19$ & $1.466(2)$ & $\mathrm{C} 18-\mathrm{H} 182$ & 0.958 \\
$\mathrm{C} 3-\mathrm{C} 4$ & $1.477(2)$ & $\mathrm{C} 18-\mathrm{H} 183$ & 0.956
\end{tabular}




\begin{tabular}{|c|c|c|c|}
\hline $\mathrm{C} 3-\mathrm{C} 14$ & $1.508(3)$ & $\mathrm{C} 19-\mathrm{C} 20$ & $1.513(3)$ \\
\hline $\mathrm{C} 3-\mathrm{H} 31$ & 0.994 & C19-H191 & 0.981 \\
\hline $\mathrm{C} 4-\mathrm{C} 5$ & $1.525(3)$ & C19-H192 & 0.991 \\
\hline $\mathrm{C} 4-\mathrm{O} 10$ & $1.448(2)$ & $\mathrm{C} 20-\mathrm{C} 21$ & $1.523(3)$ \\
\hline $\mathrm{C} 4-\mathrm{H} 41$ & 1.004 & $\mathrm{C} 20-\mathrm{O} 26$ & $1.456(2)$ \\
\hline $\mathrm{C} 5-\mathrm{O} 6$ & $1.453(2)$ & $\mathrm{C} 20-\mathrm{H} 201$ & 0.976 \\
\hline C5-H51 & 0.987 & $\mathrm{C} 21-\mathrm{O} 22$ & $1.432(2)$ \\
\hline $\mathrm{O} 6-\mathrm{C} 7$ & $1.357(3)$ & $\mathrm{C} 21-\mathrm{H} 211$ & 0.986 \\
\hline $\mathrm{C} 7-\mathrm{O} 8$ & $1.197(3)$ & $\mathrm{O} 22-\mathrm{C} 23$ & $1.363(2)$ \\
\hline $\mathrm{C} 7-\mathrm{C} 9$ & $1.493(4)$ & $\mathrm{C} 23-\mathrm{O} 24$ & $1.197(3)$ \\
\hline C9-H91 & 0.970 & $\mathrm{C} 23-\mathrm{C} 25$ & $1.490(3)$ \\
\hline C9-H92 & 0.969 & $\mathrm{C} 25-\mathrm{H} 251$ & 0.982 \\
\hline C9-H93 & 0.954 & $\mathrm{C} 25-\mathrm{H} 252$ & 0.964 \\
\hline $\mathrm{O} 10-\mathrm{C} 11$ & $1.355(2)$ & $\mathrm{C} 25-\mathrm{H} 253$ & 0.966 \\
\hline $\mathrm{C} 11-\mathrm{O} 12$ & $1.200(3)$ & $\mathrm{O} 26-\mathrm{C} 27$ & $1.355(2)$ \\
\hline $\mathrm{C} 11-\mathrm{C} 13$ & $1.491(3)$ & $\mathrm{C} 27-\mathrm{O} 28$ & $1.193(3)$ \\
\hline $\mathrm{C} 13-\mathrm{H} 131$ & 0.977 & $\mathrm{C} 27-\mathrm{C} 29$ & $1.482(3)$ \\
\hline $\mathrm{C} 13-\mathrm{H} 132$ & 0.962 & $\mathrm{C} 29-\mathrm{H} 291$ & 0.946 \\
\hline C13-H133 & 0.964 & $\mathrm{C} 29-\mathrm{H} 292$ & 0.954 \\
\hline $\mathrm{C} 14-\mathrm{O} 15$ & $1.448(2)$ & $\mathrm{C} 29-\mathrm{H} 293$ & 0.963 \\
\hline $\mathrm{C} 14-\mathrm{H} 141$ & 0.989 & & \\
\hline $\mathrm{N} 2-\mathrm{C} 1-\mathrm{C} 5$ & $106.11(15)$ & $\mathrm{O} 15-\mathrm{C} 14-\mathrm{H} 142$ & 110.3 \\
\hline $\mathrm{N} 2-\mathrm{C} 1-\mathrm{C} 21$ & $104.58(15)$ & $\mathrm{H} 141-\mathrm{C} 14-\mathrm{H} 142$ & 109.0 \\
\hline $\mathrm{C} 5-\mathrm{C} 1-\mathrm{C} 21$ & $118.30(16)$ & $\mathrm{C} 14-\mathrm{O} 15-\mathrm{C} 16$ & $114.62(16)$ \\
\hline $\mathrm{N} 2-\mathrm{C} 1-\mathrm{H} 11$ & 108.8 & $\mathrm{O} 15-\mathrm{C} 16-\mathrm{O} 17$ & $122.2(2)$ \\
\hline $\mathrm{C} 5-\mathrm{C} 1-\mathrm{H} 11$ & 109.5 & $\mathrm{O} 15-\mathrm{C} 16-\mathrm{C} 18$ & $112.17(18)$ \\
\hline $\mathrm{C} 21-\mathrm{C} 1-\mathrm{H} 11$ & 109.1 & $\mathrm{O} 17-\mathrm{C} 16-\mathrm{C} 18$ & $125.6(2)$ \\
\hline $\mathrm{C} 1-\mathrm{N} 2-\mathrm{C} 3$ & $108.97(14)$ & $\mathrm{C} 16-\mathrm{C} 18-\mathrm{H} 181$ & 108.6 \\
\hline $\mathrm{C} 1-\mathrm{N} 2-\mathrm{C} 19$ & $108.76(15)$ & $\mathrm{C} 16-\mathrm{C} 18-\mathrm{H} 182$ & 109.2 \\
\hline $\mathrm{C} 3-\mathrm{N} 2-\mathrm{C} 19$ & $116.67(16)$ & $\mathrm{H} 181-\mathrm{C} 18-\mathrm{H} 182$ & 110.2 \\
\hline $\mathrm{N} 2-\mathrm{C} 3-\mathrm{C} 4$ & $103.27(16)$ & $\mathrm{C} 16-\mathrm{C} 18-\mathrm{H} 183$ & 109.3 \\
\hline $\mathrm{N} 2-\mathrm{C} 3-\mathrm{C} 14$ & $113.92(16)$ & $\mathrm{H} 181-\mathrm{C} 18-\mathrm{H} 183$ & 110.1 \\
\hline $\mathrm{C} 4-\mathrm{C} 3-\mathrm{C} 14$ & $112.75(15)$ & $\mathrm{H} 182-\mathrm{C} 18-\mathrm{H} 183$ & 109.3 \\
\hline $\mathrm{N} 2-\mathrm{C} 3-\mathrm{H} 31$ & 111.3 & $\mathrm{~N} 2-\mathrm{C} 19-\mathrm{C} 20$ & $105.06(17)$ \\
\hline $\mathrm{C} 4-\mathrm{C} 3-\mathrm{H} 31$ & 106.4 & $\mathrm{~N} 2-\mathrm{C} 19-\mathrm{H} 191$ & 112.1 \\
\hline $\mathrm{C} 14-\mathrm{C} 3-\mathrm{H} 31$ & 108.9 & $\mathrm{C} 20-\mathrm{C} 19-\mathrm{H} 191$ & 108.8 \\
\hline $\mathrm{C} 3-\mathrm{C} 4-\mathrm{C} 5$ & $103.33(15)$ & $\mathrm{N} 2-\mathrm{C} 19-\mathrm{H} 192$ & 109.5 \\
\hline $\mathrm{C} 3-\mathrm{C} 4-\mathrm{O} 10$ & $111.18(15)$ & $\mathrm{C} 20-\mathrm{C} 19-\mathrm{H} 192$ & 111.2 \\
\hline $\mathrm{C} 5-\mathrm{C} 4-\mathrm{O} 10$ & $106.33(16)$ & H191-C19-H192 & 110.1 \\
\hline $\mathrm{C} 3-\mathrm{C} 4-\mathrm{H} 41$ & 111.5 & $\mathrm{C} 19-\mathrm{C} 20-\mathrm{C} 21$ & $101.52(15)$ \\
\hline $\mathrm{C} 5-\mathrm{C} 4-\mathrm{H} 41$ & 113.3 & $\mathrm{C} 19-\mathrm{C} 20-\mathrm{O} 26$ & $108.48(16)$ \\
\hline $\mathrm{O} 10-\mathrm{C} 4-\mathrm{H} 41$ & 110.8 & $\mathrm{C} 21-\mathrm{C} 20-\mathrm{O} 26$ & $109.03(16)$ \\
\hline $\mathrm{C} 1-\mathrm{C} 5-\mathrm{C} 4$ & $103.08(15)$ & $\mathrm{C} 19-\mathrm{C} 20-\mathrm{H} 201$ & 114.2 \\
\hline $\mathrm{C} 1-\mathrm{C} 5-\mathrm{O} 6$ & $111.65(15)$ & $\mathrm{C} 21-\mathrm{C} 20-\mathrm{H} 201$ & 113.0 \\
\hline $\mathrm{C} 4-\mathrm{C} 5-\mathrm{O} 6$ & $104.60(16)$ & $\mathrm{O} 26-\mathrm{C} 20-\mathrm{H} 201$ & 110.2 \\
\hline $\mathrm{C} 1-\mathrm{C} 5-\mathrm{H} 51$ & 111.6 & $\mathrm{C} 20-\mathrm{C} 21-\mathrm{C} 1$ & $103.69(16)$ \\
\hline $\mathrm{C} 4-\mathrm{C} 5-\mathrm{H} 51$ & 114.1 & $\mathrm{C} 20-\mathrm{C} 21-\mathrm{O} 22$ & $114.18(16)$ \\
\hline
\end{tabular}




$\begin{array}{llll}\text { O6-C5-H51 } & 111.4 & \mathrm{C} 1-\mathrm{C} 21-\mathrm{O} 22 & 110.11(16) \\ \mathrm{C} 5-\mathrm{O} 6-\mathrm{C} 7 & 116.67(17) & \mathrm{C} 20-\mathrm{C} 21-\mathrm{H} 211 & 109.2 \\ \mathrm{O} 6-\mathrm{C} 7-\mathrm{O} 8 & 123.5(2) & \mathrm{C} 1-\mathrm{C} 21-\mathrm{H} 211 & 109.9 \\ \mathrm{O} 6-\mathrm{C} 7-\mathrm{C} 9 & 110.9(2) & \mathrm{O} 22-\mathrm{C} 21-\mathrm{H} 211 & 109.6 \\ \mathrm{O} 8-\mathrm{C} 7-\mathrm{C} 9 & 125.6(2) & \mathrm{C} 21-\mathrm{O} 22-\mathrm{C} 23 & 117.04(16) \\ \mathrm{C} 7-\mathrm{C} 9-\mathrm{H} 91 & 109.0 & \mathrm{O} 22-\mathrm{C} 23-\mathrm{O} 24 & 122.9(2) \\ \mathrm{C} 7-\mathrm{C} 9-\mathrm{H} 92 & 109.0 & \mathrm{O} 22-\mathrm{C} 23-\mathrm{C} 25 & 110.27(19) \\ \mathrm{H} 91-\mathrm{C} 9-\mathrm{H} 92 & 112.0 & \mathrm{O} 24-\mathrm{C} 23-\mathrm{C} 25 & 126.8(2) \\ \mathrm{C} 7-\mathrm{C} 9-\mathrm{H} 93 & 108.5 & \mathrm{C} 23-\mathrm{C} 25-\mathrm{H} 251 & 109.0 \\ \mathrm{H} 91-\mathrm{C} 9-\mathrm{H} 93 & 108.8 & \mathrm{C} 23-\mathrm{C} 25-\mathrm{H} 252 & 109.2 \\ \mathrm{H} 92-\mathrm{C} 9-\mathrm{H} 93 & 109.4 & \mathrm{H} 251-\mathrm{C} 25-\mathrm{H} 252 & 109.7 \\ \mathrm{C} 4-\mathrm{O} 10-\mathrm{C} 11 & 116.98(16) & \mathrm{C} 23-\mathrm{C} 25-\mathrm{H} 253 & 110.1 \\ \mathrm{O} 10-\mathrm{C} 11-\mathrm{O} 12 & 122.9(2) & \mathrm{H} 251-\mathrm{C} 25-\mathrm{H} 253 & 109.6 \\ \mathrm{O} 10-\mathrm{C} 11-\mathrm{C} 13 & 110.71(19) & \mathrm{H} 252-\mathrm{C} 25-\mathrm{H} 253 & 117.07(16) \\ \mathrm{O} 12-\mathrm{C} 11-\mathrm{C} 13 & 126.35(19) & \mathrm{C} 20-\mathrm{O} 26-\mathrm{C} 27 & 122.8(2) \\ \mathrm{C} 11-\mathrm{C} 13-\mathrm{H} 131 & 108.8 & \mathrm{O} 26-\mathrm{C} 27-\mathrm{O} 28 & 12.16(18) \\ \mathrm{C} 11-\mathrm{C} 13-\mathrm{H} 132 & 110.7 & \mathrm{O} 26-\mathrm{C} 27-\mathrm{C} 29 & 112.4 \\ \mathrm{H} 131-\mathrm{C} 13-\mathrm{H} 132 & 110.5 & \mathrm{O} 28-\mathrm{C} 27-\mathrm{C} 29 & 112.8 \\ \mathrm{C} 11-\mathrm{C} 13-\mathrm{H} 133 & 107.9 & \mathrm{C} 27-\mathrm{C} 29-\mathrm{H} 291 & 108.8 \\ \mathrm{H} 131-\mathrm{C} 13-\mathrm{H} 133 & 109.0 & \mathrm{C} 27-\mathrm{C} 29-\mathrm{H} 292 & 109.4 \\ \mathrm{H} 132-\mathrm{C} 13-\mathrm{H} 133 & 109.8 & \mathrm{H} 291-\mathrm{C} 29-\mathrm{H} 292 & 106.6 \\ \mathrm{C} 3-\mathrm{C} 14-\mathrm{O} 15 & 107.24(15) & \mathrm{C} 27-\mathrm{C} 29-\mathrm{H} 293 & 106.6 \\ \mathrm{C} 3-\mathrm{C} 14-\mathrm{H} 141 & 109.4 & \mathrm{H} 291-\mathrm{C} 29-\mathrm{H} 293 & \\ \mathrm{O} 15-\mathrm{C} 14-\mathrm{H} 141 & 109.9 & \mathrm{H} 292-\mathrm{C} 29-\mathrm{H} 293 & \\ \mathrm{C} 3-\mathrm{C} 14-\mathrm{H} 142 & 111.1 & & \end{array}$

